\title{
Enjeux de la recherche sur le palmier à huile en Afrique
}

\section{Oil palm research challenges in Africa}

Oléagineux, Corps Gras, Lipides. Volume 7, Numéro 2, 138-9, Mars - Avril 2000, Dossier : Afrique, plantation et développement

\section{Auteur(s) : Jean-Claude FOLLIN}

Résumé : L'Afrique, qui fournissait $80 \%$ des exportations mondiales en 1960, a pratiquement disparu des échanges internationaux. La supériorité des rendements en Asie du Sud-Est, les coûts de production trop élevés pour un produit dont le prix, en monnaie constante, a chuté avec l'expansion des productions asiatiques, des politiques agricoles trop rigides, peu incitatives et manquant de continuité ont fortement contribué à cette chute relative1. Pourtant, l'Afrique n'est pas sans atouts, il existe des pays à fortes potentialités comme le Nigeria et le Congo et un fort engouement des petits paysans pour la culture du palmier à huile dans des pays comme la Côte d'Ivoire, le Cameroun ou même le Bénin où les conditions de culture sont pourtant considérées comme à la limite du possible.

Summary : Trade liberalization and privatization of State-owned companies have prompted a profound questioning of oil palm research in Africa. In order to ensure technology transfers to producers, research on the physical factors of production now needs to be accompanied by research in the social sciences, which is essential for understanding (a) farm economics and producer strategies; (b) producer organization and (c) the international markets and their consequences for commodity chain organization.

Keywords : oil palm research, technology transferts, social sciences.

\section{ARTICLE}

\section{Les grandes orientations de la recherche sur le palmier à huile en Afrique}

Deux grands axes s'imposent pour la recherche, dont l'importance relative varie avec les caractéristiques des exploitations concernées : la productivité des parcelles et la durabilité économique dans le respect de l'environnement.

Depuis un siècle, la rupture technologique la plus importante dans la domestication du palmier à huile a été la création de variétés hybrides, qui place le palmier à huile comme la plus productive des plantes oléagineuses. Cette recherche se poursuit et, avec les apports récents de la biologie cellulaire (notamment l'embryogenèse somatique) et moléculaire (la lecture du génome pour la sélection assistée par des marqueurs génétiques), on attend de nouveaux progrès. Cependant, la recherche d'une productivité maximale, légitime pour le système agro-industriel, doit aussi s'accompagner de la mise en marché de variétés adaptées à la culture familiale (taille des plants, résistance aux stress, composition de l'huile, etc.). Cette mise en marché doit être organisée rigoureusement et 
rapidement pour enrayer une tendance actuelle : le semis de graines issues de plants hybrides, pratique certes liée au succès du palmier à huile, mais dont les conséquences sont très préjudiciables au planteur².

L'augmentation de la productivité passe également par une meilleure compréhension du fonctionnement de la plante dans son environnement, ce qui aboutit à l'élaboration progressive de modèles, des plus simples aux plus sophistiqués, afin de calculer, dans des conditions normatives de conduite des plantations, les potentiels de production dans un site donné, de prévoir les productions annuelles et leur répartition au cours de l'année, d'adapter les densités de plantation aux composantes matériel végétal, sol et climat, d'apporter des fertilisations différenciées selon les situations et les sols, etc. Pour les petites exploitations, la modélisation apporte également de nouveaux outils pour simuler l'effet d'innovations techniques dans des systèmes de culture variés.

Une meilleure compréhension du fonctionnement de la plante en situation de production est aussi fondamentale pour la création de variétés destinées aux zones marginales, notamment pour la résistance à la sécheresse.

Se préoccuper de la durabilité de la culture c'est aborder de manière multidisciplinaire les questions qui émergent avec le vieillissement des plantations et le renouvellement de celles-ci. Quels sont les effets de la monoculture sur le long terme ? Quels diagnostics peut-on porter ? Comment caractériser l'évolution du climat, des sols, des itinéraires techniques, de la pression des maladies et des ravageurs, etc. ? Des questions d'autant plus pressantes que l'extension des cultures sur fronts pionniers arrive à son terme.

On ne possède que des réponses partielles à ces questions, et de nombreuses expérimentations sont encore nécessaires, par exemple sur :

- les types de préparation du sol ;

- la récupération des terroirs en voie de dégradation ;

- l'adaptation des itinéraires techniques aux évolutions (nouveau matériel végétal, mécanisation, etc.) ;

- l'évaluation des risques de compaction des sols ;

- I'utilisation rationnelle des fertilisants et a contrario les conséquences de leur non-utilisation, etc.

Dans le domaine de la protection des cultures, en Afrique, à l'inverse de l'Amérique du Sud où sévit la pourriture du cœur, les plantations ne sont pas menacées par des maladies foudroyantes. Seule la fusariose $^{3}$ se développe de manière régulière et pose un sérieux problème dans les replantations en zones infectées. Les travaux de création et de multiplication de variétés résistantes sont indissociables du développement du palmier à huile en Afrique.

Les ravageurs, notamment les chenilles défoliatrices et quelques coléoptères ${ }^{4}$, causent parfois des dégâts importants mais qui restent localisés. Des techniques de lutte intégrée ont été mises au point et évoluent avec les innovations ; elles permettent déjà de contrôler ces ravageurs à moindre coût.

Au-delà de la culture proprement dite, la recherche ne peut rester absente des profondes 
transformations socio-économiques qui affectent la transformation de la récolte depuis quelques années. Pendant plusieurs décennies, l'extraction de l'huile s'est faite essentiellement dans des usines installées au centre d'une zone de production, ces usines assurant la collecte dans une limite théorique estimée à $30 \mathrm{~km}$. Avec la libéralisation de la filière, cette limite théorique n'est plus respectée et de nouvelles plantations s'installent dans des régions où il n'existe pas d'huileries industrielles, ce qui, à terme, posera de graves problèmes de transformation. Par voie de conséquence, on observe la multiplication de mini-huileries artisanales, aux performances techniques souvent incertaines, mais qui répondent à une demande des producteurs. La recherche doit accompagner cette évolution, en l'évaluant du point de vue technique et économique, et en proposant des solutions diversifiées comme elle a déjà commencé à le faire avec le procédé DRUPALM d'extraction par broyage et centrifugation ${ }^{5}$, qui peut être adapté aux besoins de groupements de producteurs petits à moyens.

Enfin, des techniques doivent être proposées aux huileries industrielles ${ }^{6}$ qui doivent veiller à la qualité de leurs produits, évoluer vers plus d'efficience et moins de pollution, en satisfaisant aux exigences de la rentabilité économique et de la protection de l'environnement

\section{Implication des sciences sociales}

Les résultats de la recherche sur le palmier à huile en Afrique ont été longtemps remarquablement transférés vers les plantations industrielles et vers les plantations familiales encadrées (plantations villageoises), en fait lorsque les conditions socio-économiques de la culture étaient relativement bien maîtrisées. Cette situation n'existe plus, le libéralisme a remplacé le dirigisme des États et ces derniers interviennent de moins en moins dans l'organisation directe de la production et de la commercialisation. Les grandes plantations sont privatisées, ou en passe de l'être, et leur mode de production n'en a pas été profondément affecté. À l'inverse, à l'échelle du village, l'organisation ou la réorganisation des petits producteurs marque le pas, laissant les petits planteurs isolés face à une situation complexe.

Dans ces conditions, la recherche agricole a parfois quelques peines à se situer et les chercheurs peuvent avoir des difficultés pour cerner une demande qui s'est diversifiée et a souvent changé de nature. La demande d'amélioration de facteurs de production est toujours présente, mais elle se limite aux grandes plantations pour lesquelles le milieu est relativement bien connu et contrôlé. En dehors de ces dernières, les demandes sont rarement formulées en termes techniques et, pour être comprises, elles nécessitent la connaissance des conditions socio-économiques du milieu des producteurs.

De même, la recherche n'a plus de relais efficaces pour le transfert aux petits planteurs des innovations, le matériel végétal amélioré est plus difficile à obtenir qu'auparavant, le petit crédit n'est plus assuré et les intrants sont de moins en moins utilisés. Pour remédier à cette situation, les besoins en technologies particulières et les conditions de transfert de ces technologies demandent aussi à être mieux connues.

Les sciences sociales, sociologie et agro-économie, dans un contexte nouveau que personne n'appréhende encore vraiment, doivent jouer un rôle de plus en plus important, à la mesure des 
enjeux liés à la production et à la consommation des oléagineux en Afrique. Elles sont indispensables pour les interventions à, au moins, trois échelles :

- l'économie de l'exploitation et la stratégie des producteurs ;

- l'organisation des producteurs, niveau intermédiaire entre la production et la transformation ;

- les marchés internationaux et les conséquences sur l'organisation de la filière.

\section{Notes}

${ }^{1}$ Sur ce sujet, lire dans ce même numéro l'article de R. Hirsch.

${ }^{2}$ |l est admis que le semis de graines « tout venant » entraîne une perte de l'ordre de 40 \% (dont 25 $\%$ de plants stériles), avec un travail égal de plantation et de soins.

${ }^{3}$ Maladie de flétrissement provoquée par un champignon du sol : Fusarium oxysporum f. sp. elaeidis.

${ }^{4}$ Coléoptères géants sur les jeunes plants (Oryctes et Rhynchophorus) et mineurs de feuilles (Caelaenomedodera).

${ }^{5}$ Les modules actuels peuvent traiter de 3 à $10 \mathrm{t} / \mathrm{h}$, il est possible de " descendre " à quelques centaines de $\mathrm{kg} / \mathrm{h}$.

${ }^{6}$ En première analyse, il semble que les petites huileries de village recyclent beaucoup plus les sousproduits que les grosses unités.

\section{CONCLUSION}

En Afrique, dans le nouveau contexte du néolibéralisme, la situation actuelle de la recherche agricole est difficile. En effet, celle-ci n'a pas encore trouvé les relais lui assurant, comme par le passé, le transfert vers le milieu des producteurs de ses innovations, que ce soit dans le domaine du matériel végétal, de l'utilisation des intrants ou de la conduite des parcelles de production.

Pourtant, la recherche n'est pas sans arguments : dans la décennie à venir, l'émergence des biotechnologies et les progrès continus de l'informatique et de l'analyse spatiale provoqueront des ruptures technologiques importantes, qu'il est encore difficile de définir précisément, mais qui toucheront profondément la création variétale, la défense des cultures, la connaissance du fonctionnement de la plante et la conduite des cultures.

Ces innovations n'auront toutefois de diffusion au bénéfice du plus grand nombre des producteurs que si elles sont diversifiées et adaptées aux différents systèmes de production. Un continuum recherche-producteurs doit être assuré au travers, en particulier, d'organisations paysannes efficientes et bien gérées.

L'enjeu est de taille, l'Afrique est importatrice d'huile et ne deviendra auto-suffisante qu'à la condition d'une augmentation considérable de sa production dans les vingt années à venir. Le 
palmier à huile, de par sa productivité et la qualité de son huile, est le meilleur candidat pour assurer cette production, encore faut-il une volonté politique de développement et une recherche renouvelée dans ses approches des problèmes techniques, et des conditions socio-économiques qui les accompagnent. 